\title{
MARCAS RADULARES PRODUCIDAS POR GASTERÓPODOS PASTOREADORES DEL INTERMAREAL ROCOSO
}

\section{RADULAR MARKS PRODUCED BY GRAZING GASTROPODS OF THE ROCKY INTERTIDAL}

\author{
Yván Reyes T.'; César Córdova C.1; Leonardo Romero C. ${ }^{2}$ y Carlos Paredes Q. ${ }^{3}$
}

\section{RESUMEN}

Utilizando superficies artificiales, se pudo obtener marcas radulares de cuatro especies de gasterópodos pastoreadores comunes en las orillas rocosas de la Bahía de Ancón. En el presente trabajo se muestra las marcas radulares obtenidas en el laboratorio, del tróquido Tegula atra (Lesson, 1830), el fisurélido Fissurella peruviana Lamarck, 1822; el lótido Lottia ceciliana (Orbigny, 1841) y el litorínido Nodilittorina peruviana (Lamarck, 1822), dejadas sobre discos de cera dental. Además, se describe el tipo radular de cada especie para relacionarla con la marca producida.

Palabras clave: Pastoreo, gasterópodos, herbivoria, intermareal, cera dental, rádula.

\begin{abstract}
We had used artificial surfaces to examine the radular marks of four common grazing gastropods from Ancon Bay. In this work we show radular marks on dental wax surface of the trochid Tegula atra (Lesson, 1830), the fissurellid Fissurella peruviana Lamarck, 1822; the lottid Lottia ceciliana (Orbigny, 1841) and the littorinid Nodilittorina peruviana (Lamarck, 1822). In addition, the radular type is described to each species to relate it to the produced mark.
\end{abstract}

Key words: Grazing, gastropods, herbivory, intertidal, dental wax, radula.

\section{INTRODUCCIÓN}

Los moluscos herbívoros que habitan las orillas rocosas marinas tienen una influencia importante en las poblaciones y comunidades intermareales y submareales (Branch y Moreno, 1994; Santelices et al., 1986). De ellos, los gasterópodos pastoreadores causan efectos en la microflora epilítica (diatomeas, algas azul-verdosas, esporas, algas filamentosas microscópicas y plániulas de macroalgas) (Nicotri, 1977). Además, cabe mencionar que algunos herbívoros están involucrados en los

- Laboratorio de Ficología Marina. Facultad de Ciencias Biológicas. Universidad Nacional Mayor de San Marcos. Apartado 14-002. Lima, Perú. email: d190073@unmsm.edu.pe

${ }^{2}$ Servicio de Consultoría en Áreas Marinas. FCB. UNMSM.

${ }^{3}$ Laboratorio de Invertebrados Acuáticos. FCB. UNMSM. procesos de dispersión de propágulos de algas (Santelices, 1990). Los gasterópodos pastoreadores presentan diferentes estrategias y preferencias en su alimentación, las que dependen, entre otras cosas, de su morfología radular. Así, aquellos con rádula ripidoglosa o taenioglosa tienden a preferir en su dieta algas microscópicas y fulamentosas; mientras que los poseedores de rádula docoglosa, además de preferir algas microscópicas, también consumen algunas algas coriáceas (Steneck y Watling, 1982). Thornpson el al. (1997) describieron una metodología que emplea discos de cera dental, incrustados en rocas, para obtener marcas producto del raspado de la rádula y determinar patrones espaciales y temporales de la actividad de pastoreo de moluscos. Dicha técnica también fue utilizada por Forrest el al. (2001) para propósitos similares. El objetivo del presente trabajo fue apli- 
car esta metodología, con algunas modificaciones, para obtener en laboratorio marcas distintivas de la rádula de cuatro especies de gasterópodos de significativa abundancia relativa en la costa central del Perú: el tróquido Tegula atra (Lesson, 1830), el fisurélido Fissurella peruviana Lamarck, 1822; el lótido Lottia ceciliana (Orbigny, 1841) y el litorínido Nodilitlorina peruviana (Lamarck, 1822); y estudiar su relación con la estructura de la rádula. Este trabajo preliminar constituye una base para futuros estudios de campo sobre monitoreo de la actividad pastoreadora de gasterópodos del intermareal.

\section{MATERIAL Y MÉTODOS}

\section{Obtención de raspados en laboratorio}

Se seleccionó como sustrato cuatro rocas graníticas con un volumen aproximado de $2000 \mathrm{~cm}^{3}$, cubiertas de algas filamentosas y costrosas del intermareal de la Bahía de Ancón, Lima. Estas se trasladaron al laboratorio envueltas en papel absorbente humedecido en agua de mar y dentro de una cámara de aislación térmica a $8^{\circ} \mathrm{C}$. En el laboratorio se eliminó la.fauna presente, y con un taladro de mesa, con broca de $1,6 \mathrm{~cm}$ de diámetro, se hicieron 10 hoyos de $0,5 \mathrm{~cm}$ de profundidad distribuidos regularmente en los sustratos. Utilizando masilla epóxica se definió mejor la profundidad, forma circular y el diámetro $(1,6$ $\mathrm{cm}$ ) de los hoyos, procurando que la superficie quede plana con respecto al sustrato. Luego estos se aclimataron por separado en acuarios, con aireación continua, fotoperiodo 8:16 (luz : oscuridad) y temperatura $30: 28^{\circ} \mathrm{C}$ (día : noche), para cada una de las especies de moluscos.

Los discos de cera se obtuvieron rellenando anillos de PVC (tubo para agua caliente) de $1,6 \mathrm{~cm}$ de diámetro y $0,5 \mathrm{~cm}$ de altura, los cuales se colocaron sobre una superficie de vidrio previamente limpiada con glicerina. Para el llenado de los anillos se calento cera dental blanca de incrustación en baño maría $\left(90^{\circ} \mathrm{C}\right)$, hasta quedar completamente líquida; se vertió la cera dentro de los anillos y se la dejó enfriar hasta que recuperó su dureza. Luego se separaron los discos del vidrio. Para la parte experimental se utilizaron aquellos discos cuya superficie que estuvo en contacto con el vidrio estuviese completamente limpia, plana y sin marcas.

Se recolectaron 10 individuos de Tegula atra, Fissurella peruviana, Lotlia ceciliana y Nodilittorina peruviana; y se trasladaron al laboratorio envueltos en papel toalla humedecido dentro de una cámara de aislación térmica a $8^{\circ} \mathrm{C}$. En el laboratorio se colocaron en acuarios con aireación continua para su aclimatación.

La obtención de raspados se hizo en acuarios, por separado para las cuatro especies, colocándose en cada una de ellos una roca con 10 discos de cera y 10 individuos. Diariamente los discos fueron retirados y revisados hasta observar raspados de la rádula de los moluscos. La revisión se realizó bajo el estereoscopio, coloreando la superficie del disco con azul de metileno, a fin de contrastar los raspados y estos aparezcan más nítidos.

\section{Morfología de la rádula}

Las rádulas se sumergieron en una solución de hipoclorito de sodio al 1\%, hasta su aclaramiento, se enjuagaron en agua destilada y se conservaron en alcohol al 60\%. Para la obtención de micrografías en Microscopio Electrónico, las muestras fueron enviadas a la Universidad de Concepción, Chile. Allí se fijaron en glutaraldehido $2,5 \%$ en tampón cacodilato de sodio $0,1 \mathrm{M}$, se lavaron en tampón cacodilato de sodio $0,1 \mathrm{M}$ y se postfijaron en tetróxido de osmio al $1 \%$ en tampón cacodilato de sodio. Seguidamente las rádulas se deshidrataron en acetona de 30, 50, 70, 80, 90 y $100 \%$ por 15 minutos cada uno. Luego fueron secadas con el método de punto crítico (anhidrido carbónico líquido) y pegadas al portamuestra con pasta de plata. La muestra 

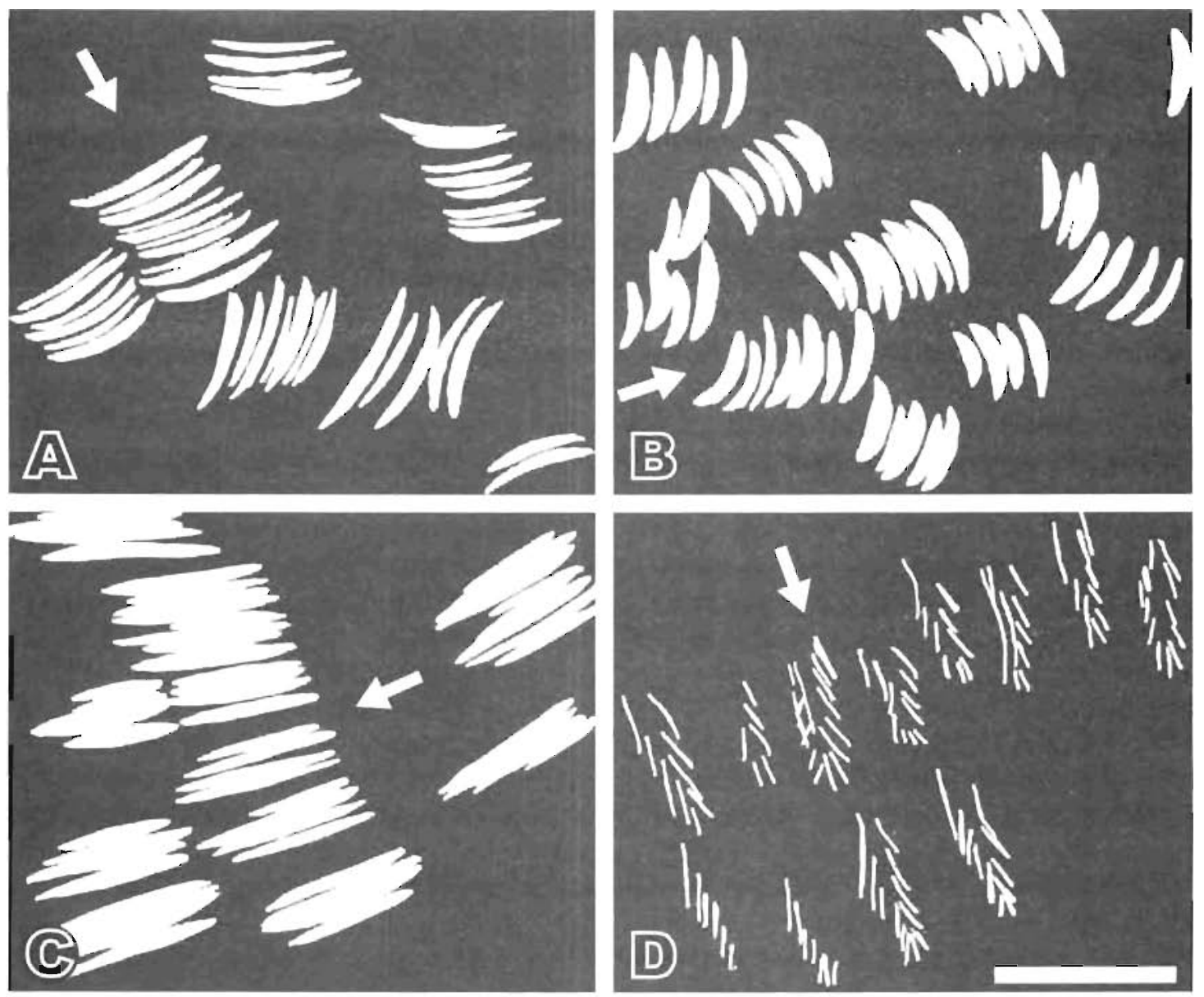

Figura 1. Esquema del patrón de marcas producidas por los raspados sobre los discos de cera dental. A) Tegula atra; B) Fissurella peruviana; C) Lottia ceciliana; y D) Nodilittorina peruviana. Las flechas indican la posible dirección que siguió el molusco al realizar los raspados. Barra $=500 \mu$

fue sombreada con un baño de oro de un espesor aproximado de $0.05 \mu$ (Anderson, 1.951) y observada en un Microscopio Electrónico de Barrido AUTOSCAN-ETEC.

\section{RESULTADOS}

\section{Raspados obtenidos en el laboratorio}

Producto del desplazamiento aleatorio durante la actividad de pastoreo sobre las rocas, se lograron registrar cuatro tipos diferentes de raspados (Fig. 1). conformados por grupos de marcas alargadas y paralelas, los cuales se distinguieron por su forma, tamaño y disposicion.
T. atra (Fig. 1.A). Raspados con un ancho aproximado de $400 \mu$ y conformados por grupos de 4-10 marcas paralelas. en forma de arco, delgadas y de extremos ahusados, con aproximadamente 30 f de grosor. cncontrăndose además marças más pequeña de manera intercalada.

E peraviana (Fig. 1.B). Raspados con un ancho aproximado de $280 \mu$ y conformados por grupos de 4-8 marcas paralelas, con forma de arco, gruesas. cortas y de extremos ahusados, con aproximadamente $40 \mu$ de grosor, encontrándose además marcas más pequenas de manera intercalada. 
L. ceciliana (Fig. 1.C). Raspados con un ancho aproximado de $150 \mu$ y conformados por grupos de 4 marcas paralelas, alargadas, rectas y con extremos ahusados, con aproximadamente $30 \mu$ de grosor. El par de marcas centrales suele ser más largo con aproximadamente $400 \mu$ de longitud.

N. peruviana (Fig. 1.D). Raspados formados por dos series de marcas oblicuas superficiales. Cada raspado con aproximadamente $400 \mu$ de longitud y $100 \mu$ de ancho. La longitud de las marcas disminuye en la dirección de desplazamiento.

Los raspados de T. atra y F. peruviana presentaron semejanzas. En ambas se notaba una curvatura similar, pero en $F$. peruviana las marcas fueron más pequeñas y más gruesas. Los raspados de $L$. ceciliana se presentaron a manera de arañazos. $N$. peruviana presentó una forma muy particular de raspado, formada por líneas diagonales casi paralelas y en dos hileras.

\section{Morfología de la rádula}

T. atra (Fig. 2.A) presenta rádula ripidoglosa. Cada hilera transversal consta de un gran diente raquidial, flanqueado a ambos lados por 5 dientes laterales y numerosos dientes marginales. El raquidial está ligeramente aplanado, con la mitad superior ensanchada en forma de media luna; además presenta un delgado segmento en el borde superior. Los laterales con la mitad superior curvada, cuyo ancho aumenta hacia los más laterales, y dentículos en el borde externo. Los dientes marginales son largos, delgados y curvados, decreciendo en tamaño hacia el margen, y presentan el borde externo serrado.

F. peruviana (Fig, 2.B) presenta rádula ripidoglosa. Cada hilera transversal consta de un diente raquidial flanqueado a ambos lados por 5 dienies laterales y numerosos dientes marginales (menos que en $T$. atra). El raquidial y los laterales son idénticos, con la porción superior curvada; el quinto lateral es conside- rablernente más grande, con la mitad superior curvada y 4 cúspides, de las cuales la segunda hacia el exterior es más grande. Los dientes marginales son largos y delgados, con la porción basal muy angosta y la mitad superior curvada; además el borde superior es curvado y ligeramente serrado.

L. ceciliana (Fig. 2.C) presenta rádula docoglosa. En cada hilera transversal posee 3 pares de dientes laterales y un par de marginales (uncini), no hay raquidial. Los dientes laterales son grandes y curvados, con una amplia porción basal y el borde superior agudo. Los dientes del primer par lateral son los más largos y centrales, unidos por una porción central. Los dientes del segundo par son más cortos y están ubicados por debajo del primer par. El tercer par es más pequeño y de posición lateral en relación al segundo par. Los dientes marginales son curvados, aplanados y muy pequeños.

N. peruviana (Fig. 2.D) presenta rádula taenioglosa, donde cada hilera transversal consta de un diente raquidial flanqueado a ambos lados por 2 dientes laterales y un marginal. El raquidial es pequeño y ligeramente curvado, con la porción basal amplia y 3 cúspides de las cuales la central es la más grande. Los dientes laterales son gruesos, con la mitad superior curvada y ancha, con 3 cúspides, siendo mayor la central. El primer par de laterales presenta lá cúspide central de forma rectangular. Los dientes marginales son gruesos, en forma de garra, con la mitad superior curvada y 9 cúspides similares; además presenta procesos basales, lateralmente y a manera de alas, siendo más grande la del borde externo.

\section{DISCUSIÓN}

El patrón de marcas en forma de arco obtenidos para $T$. atra y $F$, peruviana coinciden con aqueilas obtenidas por Thompson et al. (1997) para especies con rádula ripidoglosa (Calliostoma zizyphinum y Gibbula spp.). Por otro lado, Steneck y Watling (1982) mencio- 

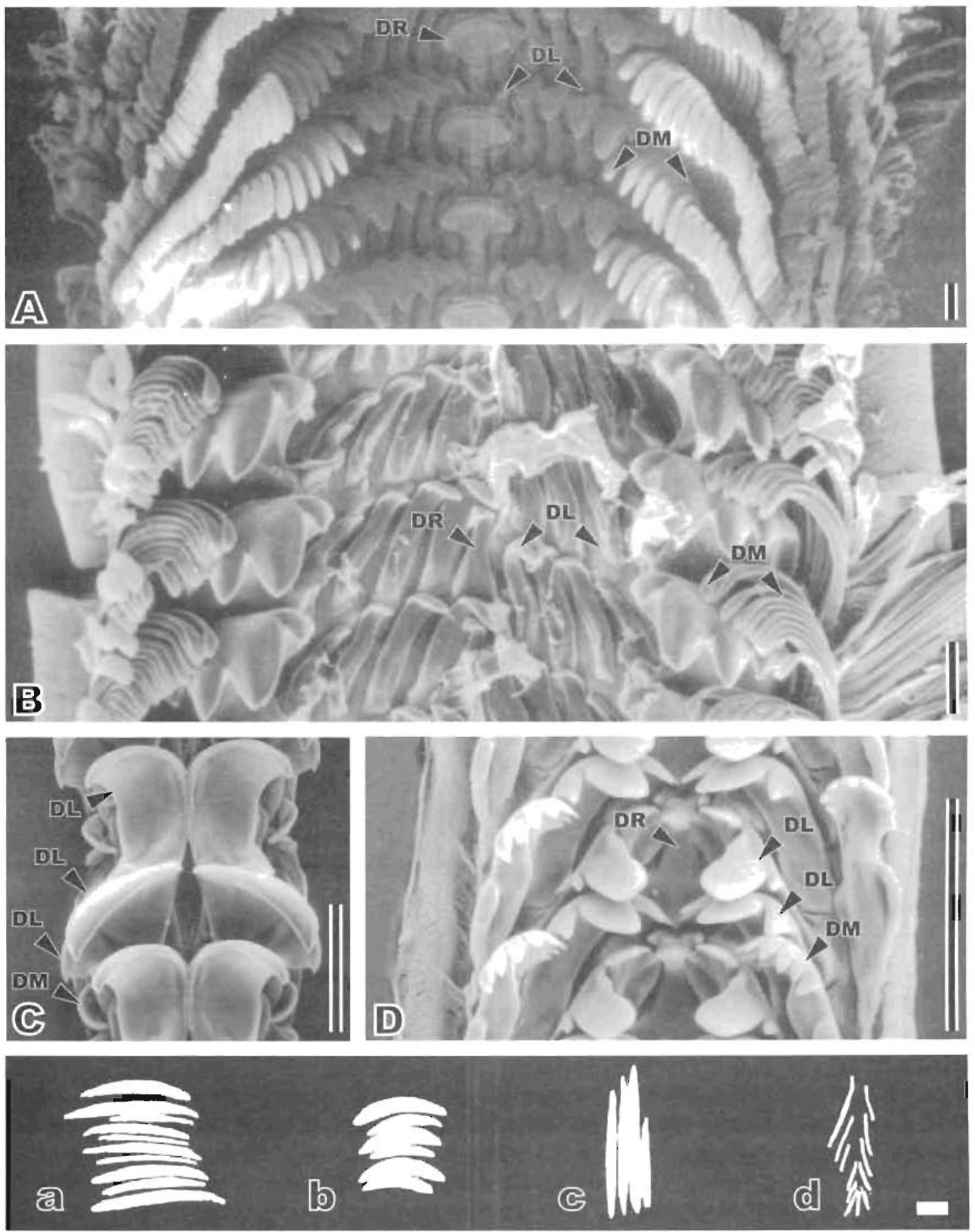

Figura 2. Micrografías a Microscopio Electrónico de Barrido de la rádula (mayúscula) y esquemas de las marcas producidas sobre los discos de cera dental (minusculas). A ya. Tegula atra; B y b. Fissurella peruviana; C y c. Lottia ceciliana; y D y d. Nodilittorina peruviana. DR, diente raquidial; $\mathrm{DL}$, diente lateral; DM, diente marginal. Barra $=100 \mathrm{H}$ 
nan que las rádulas ripidoglosas funcionan a manera de escoba por la presencia de los numerosos dientes marginales en cada hilera, los cuales al extenderse amplían el área de acción, dando como resultado numerosos puntos de contacto entre la rádula y el sustrato. En $T$. atra y $F$. peruviana el ancho de la rádula es mayor que el de los raspados, lo que nos indica que sólo una porción de la hilera de dientes penetra el sustrato. El ancho del raspado es menor en $F$. peruviana, pero el grosor de las marcas es mayor, lo que podría deberse a una menor área de contacto y a una mayor presión ejercida por los dientes sobre la cera.

Thompson et al. (1997) y Forrest et al. (2001) muestran que el patrón de raspado de rádulas docoglosas de gasterópodos patélidos consiste en arañazos irregulares sobre la cera dental. Para el caso del lótido L. ceciliana los raspados en forma de arañazos son mucho más regulares, mostrando 4 marcas en cada uno, probablemente por la acción de las cúspides del primer y segundo par de dientes laterales. La rádula de L. ceciliana presenta sólo 3 pares de dientes laterales y un par de marginales de tamaño reducido (Gallosa, 1975), mientras que el patélido $P$. vulgata presenta un número mayor de laterales y marginales (Hyman, 1967; Ridgway et al., 1998), lo cual justificaría la diferencia de marcas entre estas dos familias.

El patrón de raspado para $N$. peruviana, gasterópodo con rádula taenioglosa, consiste en dos series de marcas oblicuas superficiales producidas posiblemente cuando los dientes convergen hacia el centro durante la retracción (Steneck y Watling, 1982). Thompson et al. (1997) y Forrest et al. (2001) muestran adicionalmente marcas paralelas presentes en los raspados de los litorínidos Littorina obtusata y Bembicium nanum respectivamente.

Consideramos que para una mejor descripción de las relaciones entre la forma de las marcas producidas en los raspados y la estructura de la rádula quedan por determinar con exactitud la forma de acción de la misma so- bre la cera, así como la dirección que sigue el molusco al realizar los raspados.

A pesar de la adaptación realizada a la técnica descrita por Thompson et al. (1997), se logró obtener marcas distintivas de la rádula de cuatro especies de gasterópodos de significativa abundancia relativa en la costa central del Perú.

\section{AGRADECIMIENTOS}

Los autores agradecen el apoyo logístico brindado por el grupo de investigación DePSEA, quienes además facilitaron la realización de un ensayo de campo. Agradecemos también a los integrantes del Laboratorio de Microscopía Electrónica, Dirección de Investigación de la Universidad de Concepción, quienes de manera desinteresada se encargaron de todo lo relacionado al procesamiento de las muestras para Microscopía Electrónica de Barrido y obtención de micrografías.

\section{LITERATURA CITADA}

Anderson, T. 1951. Techniques for the preservation of three-dimensional structures in preparing specimens for the electron microscope. Trans. N. Y. Acad. Sci., Ser. II. 13: 130-134.

Branch, G. \& C. Moreno. 1994. Intertidal and Subtidal Grazers, In: W. Siegfried (Ed.). Rocky Shores: Exploitation in Chile and South Africa. Ecological Studies 103. Springer-Verlag, pp. $75-100$.

Forrest, R.; M. Chapman \& A. Underwood. 2001. Quantification of radular marks as a method for estimating grazing of intertidal gastropods on rocky shores. J. Exp. Mar. Biol. Ecol, 258: 155-171.

Gallosa, G. 1975. Morfología radular en especies de la familia Acmaeidae (Gastropoda, Aspidobranchia). Tesis para optar el grado de Bachiller. Facultad de Ciencias Biológicas, UNMSM, Lima-Perú.

Hyman, L. 1967. The Invertebrates. Vol 6. Mollusca 1, McGraw-Hill, New York Book Company.

Nicotri, M. 1977. Grazing effects of four marine intertidal herbivores on the microflora. Ecology, 58: 1020-1032.

Ridgway, S.; D. Reid; J. Taylor; G. Branch \& A. Hodgson. 1998. A cladistic phylogeny of the family Patellidae (Mollusca: Gastropoda). 
Philos. Trans. R. Soc. Lond. (Biol.), 353: 1645-1671.

Santelices, B. 1990. Pattems of reproduction dispersal and recruitment in seaweeds. Oceanogr. Mar. Biol. Ann. Rev., 28: 177-276.

Santelices, B.; J. Vasquez \& I. Meneses. 1986. Patrones de distribución y dietas de un gremio de moluscos herbívoros en hábitats intermareales expuestos de Chile central. Monografías Biológicas, Universidad Católica de Chile-San- tiago, 4: 147-17!.

Steneck R. \& L. Watling. 1982. Feeding capabilities and limitation of herbivorous molluscs: A functional group approach. Mar. Biol., 68: 299-319.

Thompson, R.; L. Johnson \& S. Hawkins. 1997. A method for spatial and temporal assessment of gastropod grazing intensity in the field: the use of radula scrapes on wax surfaces. J. Exp. Mar. Biol. Ecol., 218: 63-76. 